\title{
LEMIERRE SYNDROME: A RARE CASE OF FORGOTTEN DISEASE: A CASE REPORT
}

\author{
S. K. Kanaujia ${ }^{1}$, Ashutosh Singh ${ }^{2}$, Shivani Nautiyal ${ }^{3}$, Kumar Ashutosh ${ }^{4}$
}

\section{HOW TO CITE THIS ARTICLE:}

S. K. Kanaujia, Ashutosh Singh, Shivani Nautiyal, Kumar Ashutosh. "Lemierre Syndrome: A rare case of Forgotten Disease". Journal of Evolution of Medical and Dental Sciences 2014; Vol. 3, Issue 46, September 22; Page: 11283-11287, DOI: $10.14260 /$ jemds/2014/3471

ABSTRACT: Lemierre Syndrome: A rare case of forgotten desease. Lemierre's syndrome is a rare condition characterized by septic thrombophlebitis of the internal jugular vein and metastatic abscesses following or pharyngeal infection. Though classically caused by Fuso bacterium necrophorum, a number of other causative organisms have been reported in literature. We report a case of lemierre's syndrome following pharyngitis.

KEYWORDS: Internal jugular vein thrombosis, Lemierre's syndrome, thrombophlebitis.

INTRODUCTION: Lemierre's syndrome is a septic thrombophlebitis of the internal jugular vein (IJV) commonly caused by anaerobic oropharyngeal flora that is usually followed by fulminant sepsis and rapid death in the pre-antibiotic era. Lemierre's syndrome (necrobacillosis or postanginal septicaemia) was first reported in 1900 by Gourmont and Cade $^{1}$ and later described by Andre Lemierre in 1936 in a review of 20 cases. $^{2}$ It is characterized by oropharyngeal infection, thrombophlebitis of internal jugular vein (IJV) and metastatic abscesses. It is classically due to anaerobic infection and Fuso bacterium necrophorum is the most common pathogen ${ }^{3}$. In the preantibiotic era this syndrome was common and usually fatal. With the advent and widespread use of antibiotics this is rarely reported and became the "forgotten disease." 4

CASE REPORT: A 16 year-old young male with no co-morbidities presented with history of sore throat of three days duration associated with difficulty in swallowing, one day before presenting to the hospital, he developed pain and progressive swelling on the both side of the neck and back. On examination of oral cavity there was congestion of posterior pharyngeal wall suggestive of pharyngitis.

He was afebrile and had a tender swelling extending from angle of mandible to the areola of chest and in the back between scapulas. Further systemic examination was unremarkable and his Hb9.6 gm\%, total count of 7800 cells/cumm, platelet count 286000 cells/cumm, ESR $15 \mathrm{~mm} / \mathrm{h}$ and was HIV negative. His USG neck shows that right internal jugular vein was engorged; thick walled, non-compressible, lumen was filled with echogenic thrombus. No flow was detected in color Doppler.

Left internal jugular vein showed narrowing of lumen with thickened in whole length more marked in lower half. Upper half showed turbulent blood flow on color Doppler lower half showed minimal internal flow. His CT scan neck with thorax shows venous thrombosis of right axillary, sub clavian veins and superior venacava with back flow venous changes. Multiple enlarged lymphadenopathies in both cervical region, root of neck, axillary and mediastinal planes.

DISCUSSION: In 1936, Andre Lemierre published a series of 20 cases of throat infections with anaerobic septicemia, of which 18 died. Following the introduction of antibiotics in 1940s, and its 
widespread use for streptococcal pharyngitis, the incidence of Lemierre's syndrome has fallen dramatically to a degree that it had been called the forgotten disease More recently though, there has been a rapid increase in the reporting of Lemierre's syndrome.one could assume that this rapid increase may be due to increased antibiotic resistance or change in prescription pattern. ${ }^{5}$

Fuso bacterium nucleatum and Fuso bacterium necrophorum are the most commonly isolated from patients of lemierres syndrome. Fuso bacterium species are normal inhabitants of the oral cavity, the female genital tract, and the gastrointestinal tract. Of this species, F nucleatum and F necrophorum are the most commonly isolated. They are slow growing, strictly anaerobic, gramnegative pleomorphic bacilli. F necrophorum, the most common pathogen, has an ability to invade as a primary pathogen.

This feature is related to the bacteria's ability to produce lipopolysaccharide endotoxin, leukocidin, and haemolysin. Other organisms isolated from patients with this syndrome include Bacteroides, Streptococcus, Peptostreptococcus and Eikenella corrodens, with more than one pathogen being reported in a few cases ${ }^{6}$. The primary source of infection is usually the palatine tonsils, with most patients presenting with exudative tonsillitis or peritonsillar abscesses. ${ }^{7}$

The development of thrombophlebitis of the IJV is associated with neck pain and swelling. This may occasionally be associated with trismus, otalgia and dysphagia. ${ }^{8}$ Internal jugular venous thrombosis that results from the adjacent inflammatory process or extension from the tonsillar veins acts as a nidus of infection that may spread hematogenously and result in septicemia and septic embolization, which occur most commonly in the lungs. ${ }^{9}$

In a recent literature review, $97 \%$ of 38 patients had evidence of pleuropulmonary septic emboli, and 15\% developed septic arthritis. ${ }^{9}$ Hepatic and splenic abscesses, osteomyelitis, meningitis, epidural abscess, and diffuse encephalopathy are also described in association with this syndrome. ${ }^{9}$ The major clinical features of Lemierre's syndrome include primary infection of the oropharynx, bacteremia, radiographic or clinical evidence of internal jugular vein thrombosis, and septic metastatic foci. Accurate history and physical examination are needed for diagnosis.

The syndrome should be suspected in young, otherwise healthy patients who have an underlying oropharyngeal infection and follow a worsening clinical course, requiring hospitalization for sepsis and worsening pulmonary symptoms in the setting of a recent pharyngeal infection. The patient's lateral neck swelling and tenderness (representing thrombophlebitis of the internal jugular or surrounding veins) is often mistaken for cervical lymphadenopathy.

Patients can also demonstrate the "cord sign", an induration of internal jugular vein beneath the anterior border of the sternocleidomastoid muscle 9 . Other manifestations can include localized arthralgias and diffuse abdominal pain, which may represent septic embolic seeding of joints and abdominal micro abscesses. The hip is the most commonly infected joint in published series, but osteomyelitis in Lemierre's syndrome is rare. Abnormal coagulation studies and liver function tests, in the situation of hepatic seeding and abscess formation, may also be seen. ${ }^{10}$

Imaging of internal jugular vein and associated vasculature may be accomplished with ultrasound, computed tomography, magnetic resonance imaging to establish presence of thrombophlebitis. Contrast computed tomography of the neck provides definitive diagnosis, showing distended veins with enhancing walls, intraluminal filling defects, and swelling of adjacent soft tissues. ${ }^{11}$ Ultrasonography can also confirm internal jugular vein thrombosis, showing localized echogenic regions within a dilated vessel. 
Our patient presented with painful neck swelling which progressed to upper chest in short duration of two days. There was history of pain in throat and difficulty in swallowing from three days prior to presentation in our opd suggestive of acute pharyngitis which was later on confirmed on oropharynx examination which revealed congestion on posterior pharyngeal wall and tonsillar fossa. We immediately started empirical broad spectrum antibiotic therapy. USG and CECT of neck and thorax were done.

Thrombosis of internal jugular vein in USG and extensive thrombosis of internal jugular vein, sub clavian vein and superior vena cava added to our clinical diagnosis of lemierre's syndrome. We did not get any cultures of pathogen though in throat swab may be due to early initiation of antibiotic therapy.

The recommended treatment of Fuso bacterium species in Lemierre's syndrome is combination therapy with parenteral high dose penicillin and metronidazole. Intravenous clindamycin may be used in penicillin-allergic patients ${ }^{12}$. Pulmonary abscess and empyema must be addresses with definitive surgical drainage and evacuation. The role of anticoagulant therapy is controversial and is not presently recommended as a standard of care. ${ }^{13}$

CONCLUSION: The advent and widespread use of antibiotics has made this syndrome a rarity and is frequently overlooked. Otolaryngologist should be aware of the syndrome in patients with oropharyngeal infection who later present with signs of systemic illness or pulmonary involvement. A high degree of clinical suspicion is necessary for diagnosis. It is important to recognize it early and initiate adequate treatment to reduce morbidity and prevent mortality.

\section{REFERENCES:}

1. Golpe R, Marin B, Alonso M. Eponyms in medicine revisited lemierre's syndrome (necro bacillosis) Postgrad Med J. 1999; 75: 141-4.

2. Lemierre A. On certain septicaemias due to anaerobic organisms. Lancet. 1936; 1: 701-3.

3. Krishna K, Diwan AG, Gupt A. Lemierre's syndrome: The syndrome quite forgotten. J Assoc Physicians India.2012; 60: 60-3.

4. Lu MD, Vasavada Z, Tanner C. Lemierre syndrome following oropharyngeal infection: A case series. J Am Board Fam Med. 2009; 22: 79-83.

5. Peter D. Karkos, AFRCS, MPhil; Sheetal Asrani, MBBS; Christos D. Karkos, FRCS, PhD; Samuel C. Leong, M.R.C.S. Ed, DO-HNS; Evangelia G. Theochari, MD; Thalia D. Alexopoulou, MD; Assimakis D. Assimakopoulos: Lemierre's Syndrome: A Systematic Review. Laryngoscope, 119:15521559, 2009.

6. Alherabi A: A case of Lemierre syndrome. Ann Saudi Med. 2009 Jan-Feb; 29(1): 58-60.

7. SubiranaPozo FX, Carreras S J, Lorente Guerrero J, Crego de Pablos F, Garcia Lopez M, Grasa Perez J, et al. Lemiere's syndrome. Thromb bophlebitis of the internal jugular vein induced by oropharyngeal lesion: a case report. An OtorrinolaringolIbero Am. 2001; 28(4): 419-30.

8. Clarke MG, Kennedy NJ, Kennedy K. Serious consequences of a sore throat. Ann R Coll Surg Engl. 2003; 85(4): 242-4.

9. Sinave CP, Hardy GJ, Fardy PW. The Lemierre syndrome: suppurative thrombophlebitis of the internal jugular vein secondary to oropharyngeal infection. Medicine 1989; 68: 85-94. 
10. Hagelskjaer LH, Prag J, et al.: Incidence and clinical epidemiology of necro bacillosis in Denmark 1990-1995.Eur J Clin Microbiol Infect Dis 1998, 17: 561-565.

11. Goyal M, Sharma R, Jain Y, et al.: Unusual radiological manifestations of Lemierre's syndrome: a case report. Pediatr Radiol 1995, 15(1): S105-S106.

12. Hagelskjaer KL, Prag J: Human necrobacillosis, with emphasis on Lemierre's syndrome. Clin Infect Dis 2000, 31: 524-532.

13. Stahlman GC, DeBoer DK, Green NE: Fuso bacterium osteomyelitis and pyarthrosis: a classic case of Lemierre's syndrome. J Pediatr Orthop 1996, 16:529-532.

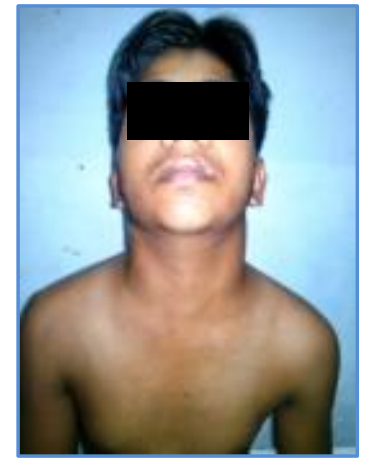

Fig. 1

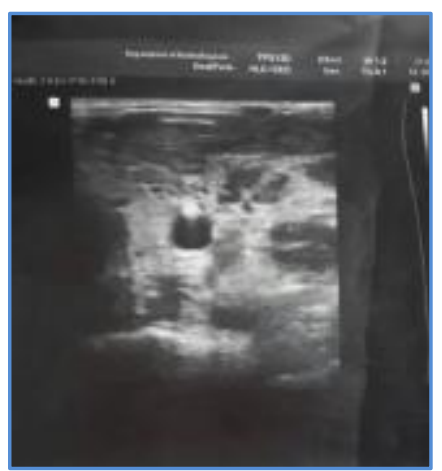

Fig. 3

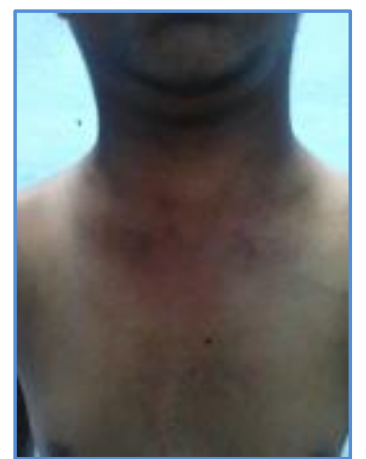

Fig. 2

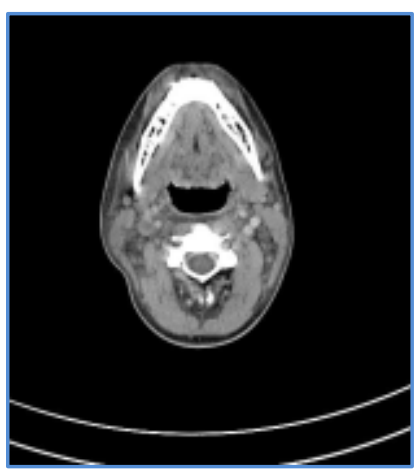

Fig. 4

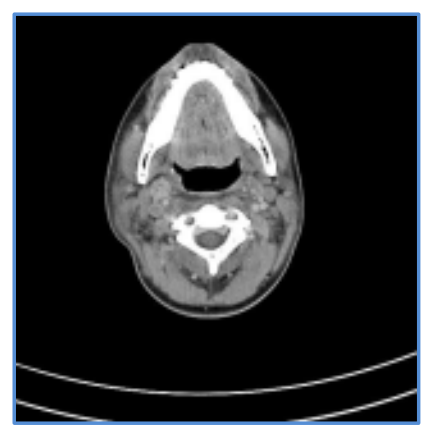

Fig. 5 


\section{AUTHORS:}

1. S. K. Kanaujia

2. Ashutosh Singh

3. Shivani Nautiyal

4. Kumar Ashutosh

\section{PARTICULARS OF CONTRIBUTORS:}

1. Assistant Professor \& HOD, Department of Otorhinolaryngology (ENT), G.S.V.M.

Medical College, Kanpur.

2. Junior Resident, Department of Otorhinolaryngology (ENT), G.S.V.M. Medical College, Kanpur.

3. Junior Resident, Department of Otorhinolaryngology (ENT), G.S.V.M. Medical College, Kanpur.
4. Junior Resident, Department of Otorhinolaryngology (ENT), G.S.V.M. Medical College, Kanpur.

\section{NAME ADDRESS EMAIL ID OF THE}

\section{CORRESPONDING AUTHOR:}

Dr. Ashutosh Singh,

Room No. 52,

P. G. Boys Hostel, G.S.V.M. Medical College,

Kanpur-208002.

Email: ashuenrapt@gmail.com

Date of Submission: 03/09/2014.

Date of Peer Review: 04/09/2014.

Date of Acceptance: 16/09/2014.

Date of Publishing: 22/09/2014. 